\title{
Estudo Morfométrico Comparativo de 58 Populações Brasileiras de Rotylenchulus reniformis (Nemata: Rotylenchulinae)*
}

\author{
Pedro L. M. Soares ${ }^{1}$, Jaime M. dos Santos $^{2} \&$ Antonio S. Ferraudo 3 \\ 1,2Departamento de Fitossanidade; ${ }^{3}$ Departamento de Ciências Exatas, Faculdade de Ciências Agrárias e Veterinárias - UNESP, \\ Campus de Jaboticabal, CEP 14884-900, Jaboticabal, SP, fone/fax: (016) 3202-3641, e-mail: pedrolms@ hotmail.com
}

(Aceito para publicação em 19/04/2004)

Autor para correspondência: Pedro L. M. Soares

SOARES, P.L.M., SANTOS, J.M. \& FERRAUDO, A.S. Estudo morfométrico comparativo de 58 populações brasileiras de Rotylenchulus reniformis (Nemata: Rotylenchulinae). Fitopatologia Brasileira 29:419-424. 2004.

\section{RESUMO}

Foram recuperadas 58 populações de Rotylenchulus reniformis de amostras de solo e raízes de diferentes culturas e inoculadas em plantas de algodoeiro (Gossypium hirsutum) cv. COODETEC 402 e de mamona (Ricinus communis), mantidas em vasos de argila em casa de vegetação do Departamento de Fitossanidade da UNESP/FCAV, Campus de Jaboticabal - São Paulo. Foi realizado estudo morfométrico comparativo das populações ao microscópio óptico composto, seguido da ordenação das populações segundo análises multivariadas de agrupamento e de componentes principais. Foram avaliadas 11 variáveis morfométricas em dez fêmeas jovens de cada população e sete variáveis derivadas. A amplitude de variação de caracteres morfométricos em populações brasileiras desse nematóide tais como, comprimento do estilete, $\mathrm{V}$ e forma da cauda, é maior que em populações da mesma espécie de outras regiões do mundo. Os dados obtidos confirmam que o comprimento do estilete, presença de machos e V são suficientes para identificação de $R$. reniformis e que esta é a espécie do grupo predominante nos agroecossistemas brasileiros.

Palavras-chave adicionais: Taxionomia, nematóide reniforme, análise multivariada, morfometria.

\section{ABSTRACT}

Comparative morphometric study of 58 Brazilian populations of Rotylenchulus reniformis (Nemata: Rotylenchulinae)

Fifty-eight different populations of Rotylenchulus reniformis were recovered from soil and root samples of different crops and inoculated in cotton (Gossypium hirsutum) cv. COODETEC 402 and castor bean (Ricinus communis) in clay pots in the greenhouse at the Departamento de Fitossanidade of the Universidade Estadual Paulista, Jaboticabal Campus, São Paulo State, Brazil. Using a light microscope, a comparative morphometric study was carried out which

\section{INTRODUÇÃO}

De acordo com Robinson et al. (1997), os registros mundiais de hospedeiros de Rotylenchulus reniformis Linford \& Oliveira, 1940, eram de 314 espécies de plantas de 77 famílias e os de não hospedeiros somavam apenas 74 espécies, das quais 22 estavam incluídas nos dois grupos.

Significativas variações morfológicas e biométricas em seis populações de $R$. reniformis de áreas geográficas e hospedeiros distintos foram encontradas na Índia (PrasadaRao et al., 1998). Alta variabilidade em relação à forma da região labial e cauda de fêmeas imaturas e machos, e a forma do corpo e projeção terminal de fêmeas maduras foi observada nessas populações. O comparativo entre as populações revelou diferenças significativas em relação ao comprimento e largura

\footnotetext{
*Parte da Dissertação de Mestrado em Agronomia do primeiro autor (2003), desenvolvido com Auxílio à Pesquisa da FAPESP (Processo No. 01/08127-2).

${ }^{1}$ Bolsista do CNPq
}

included a multivariate analysis of all the populations. Eleven morphometric and seven derived variables were evaluated in ten young females of each population. The range of morphometric values and variables in the Brazilian populations of this nematode such as length of the stylet, $\mathrm{V}$ and tail shape are wider than in populations of the same species from others regions of the world. The data confirmed that the length of the stylet, presence of males and $\mathrm{V}$ are sufficient for identification of $R$. reniformis. This also is the predominated species of the group found in Brazilian agro-ecosystems. do corpo, distância do orifício da glândula dorsal esofagiana à base do estilete, distâncias da extremidade anterior ao bulbo mediano do esôfago e ao poro excretor e o comprimento da porção hialina da cauda em fêmeas jovens. Concluíram que uma população obtida de videira (Vitis spp.) era morfologicamente distinta de todas as outras. Ganguly \& Ramesh (1996) mostraram a influência do hospedeiro sobre variáveis morfométricas, tais como comprimento e largura do corpo, a proporção "a" e o comprimento da projeção terminal de fêmeas adultas. A variável V (\%) não foi influenciada. Na opinião dos autores, o hospedeiro influenciou marcadamente o hábito de parasitismo do nematóide e aumentou a amplitude de variação da maioria das variáveis estudadas em fêmeas adultas e ovos, comparadas a espécimes topotipos de $R$. reniformis. Sudershan et al. (1997) também constataram a influência do hospedeiro sobre a variabilidade morfométrica intra-específica em $R$. reniformis.

A análise de agrupamentos aplicada às variáveis morfométricas ou derivadas, tais como L, a, b, c, c', h, V, T, o, 
comprimento do estilete e dos espículos, obtidas de fêmeas vermiformes, machos e juvenis de segundo estádio, foi efetuada por Suyadi (1997) em populações não identificadas do gênero Rotylenchulus coletadas na Estação Experimental da Universidade das Filipinas em Los Banos. Os resultados indicaram que todas as populações examinadas pertenciam a R. reniformis.

Preferência por hospedeiros diferentes, habilidade de se reproduzirem em mamona (Ricinus communis L.), algodoeiro (Gossypium hirsutum L.) e soja [Glycine max (L.) Merril] e presença ou ausência de machos na população têm sido relatadas entre populações de Rotylenchulus spp. em diferentes regiões do mundo (Dasgupta \& Seshadri, 1971; Nakasono, 1983; McGawley \& Overstreet, 1995).

Em estudo morfológico de 48 populações recuperadas de solos cultivados com abacaxizeiro [Annona comosus (L.) Merr], algodoeiro, feijoeiro (Phaseolus vulgaris L.), mamoeiro (Carica papaya L.), maracujazeiro (Passiflora edulis Sims.), soja e tomateiro (Lycopersicon esculentum Mill.), a maior parte dos estados de São Paulo (32 populações) e Góias (12), algumas da Bahia (1), Espírito Santo (1) e Mato Grosso (2), Soares et al. (2003) determinaram todas como R. reniformis. Verificaram que a amplitude de variação de caracteres morfológicos analisados, tais como, comprimento do estilete, $\mathrm{V}(\%)$ e forma da cauda, era maior do que a registrada para as populações de outras regiões do mundo.

Este trabalho teve como objetivo ampliar o estudo da variabilidade morfométrica de Rotylenchulus spp., com 58 populações brasileiras obtidas de solo de 13 diferentes cultivos, comparando-as segundo análises multivariadas de agrupamentos e de componentes principais.

\section{MATERIAL E MÉTODOS}

\section{Organização da coleção das populações de Rotylenchulus spp.}

Cinqüenta e oito populações de Rotylenchulus spp. foram recuperadas de amostras de solo e raízes de diferentes culturas e regiões (Tabela 1) e inoculadas em plantas de algodoeiro cv. COODETEC 402 e de mamona mantidas em vasos de argila contendo substrato tratado, em casa de vegetação do Departamento de Fitossanidade da UNESP/ FCAV, Campus de Jaboticabal. Os demais procedimentos foram descritos por Soares et al. (2003).

\section{Mensurações das populações de Rotylenchulus spp. e análise dos dados}

Espécimes de Rotylenchulus spp. foram preparadas para exame ao microscópio ótico composto conforme metodologia descrita por Soares et al. (2003). As 3.480 fotomicrografias dos espécimes inteiros e partes do corpo de fêmeas foram documentadas de modo a permitir as determinações das seguintes variáveis (1 a 11) em micrômetros $(\mu \mathrm{m})$ e proporções (12 a 18), perfazendo um total de 6.380 mensurações de todas as populações de Rotylenchulus spp. estudadas. Foram consideradas as seguintes variáveis: 1)
TABELA 1 - Coleção das populações de Rotylenchulus spp. utilizadas no estudo, mantidas em casa de vegetação do Departamento de Fitossanidade da UNESP

\begin{tabular}{|c|c|c|}
\hline $\begin{array}{l}\text { Identificação } \\
\text { da população }\end{array}$ & $\begin{array}{c}\text { Município e UF } \\
\text { de origem }\end{array}$ & $\begin{array}{c}\text { Cultura de } \\
\text { origem }\end{array}$ \\
\hline 1 & Sta Helena - GO & Não consta \\
\hline 2 & Itabuna - BA & Não consta \\
\hline 3 & Jaboticabal - SP & Meloeiro ${ }^{\text {a }}$ \\
\hline 4 & Ituverava - SP & Algodoeiro ${ }^{b}$ \\
\hline 5 & Ituverava - SP & Algodoeiro \\
\hline 6 & Jaborandi - SP & Algodoeiro \\
\hline 7 & Jaborandi - SP & Algodoeiro \\
\hline 8 & Jaborandi - SP & Algodoeiro \\
\hline 9 & Sta Helena - GO & Algodoeiro \\
\hline 10 & Sta Helena - GO & Algodoeiro \\
\hline 11 & Castelândia - GO & Algodoeiro \\
\hline 12 & Edéia - GO & Algodoeiro \\
\hline 13 & Edéia - GO & Algodoeiro \\
\hline 14 & Porteirão - GO & Soja ${ }^{c}$ \\
\hline 15 & Porteirão - GO & Sorgo ${ }^{d}$ \\
\hline 16 & Porteirão - GO & Algodoeiro \\
\hline 17 & Porteirão - GO & Algodoeiro \\
\hline 18 & Porteirão - GO & Algodoeiro \\
\hline 19 & Porteirão - GO & Soja \\
\hline 20 & Porteirão - GO & Soja \\
\hline 21 & Porteirão - GO & Soja \\
\hline 22 & Porteirão - GO & Algodoeiro \\
\hline 23 & Itumbiara - GO & Soja \\
\hline 24 & Porteirão - GO & Soja \\
\hline 25 & Itumbiara - GO & Algodoeiro \\
\hline 26 & Itumbiara - GO & Algodociro \\
\hline 27 & Porteirão - GO & Soja \\
\hline 28 & Porteirão - GO & Soja/Milheto ${ }^{\mathrm{c}}$ \\
\hline 29 & Porteirão - GO & Soja \\
\hline 30 & Buritizal - SP & Milho ${ }^{f}$ \\
\hline 31 & Buritizal - SP & Milho \\
\hline 32 & Eunapólis - BA & Pinha $\mathrm{g}$ \\
\hline 33 & Eunapólis - BA & Pinha \\
\hline 34 & Porteirão - GO & Algodoeiro \\
\hline 35 & Porteirão - GO & Algodoeiro \\
\hline 36 & Riolândia - SP & Não consta \\
\hline 37 & Ituverava - SP & Algodoeiro \\
\hline 38 & Jaboticabal - SP & Tomateiro $\mathrm{h}$ \\
\hline 39 & Jaborandi - SP & Algodoeiro \\
\hline 40 & Jaborandi - SP & Algodoeiro \\
\hline 41 & Miguelópolis - SP & Algodoeiro \\
\hline 42 & Miguelópolis - SP & Algodoeiro \\
\hline 43 & Taiaçu - SP & Citros ${ }^{i}$ \\
\hline 44 & Taiaçu - SP & Citros \\
\hline 45 & Taiaçu - SP & Citros \\
\hline 46 & Araraquara - SP & Cenoura $^{j}$ \\
\hline 47 & Frutal - MG & Feijoeiro $^{k}$ \\
\hline 48 & Monte Alto - SP & Pasto \\
\hline 49 & Miguelópolis - SP & Soja \\
\hline 50 & Miguelópolis - SP & Soja \\
\hline 51 & Riolândia - SP & Algodoeiro \\
\hline 52 & Jaboticabal - SP & Tomateiro \\
\hline 53 & Jaboticabal - SP & Mamoeiro 1 \\
\hline 54 & Guaira - SP & Algodoeiro \\
\hline 55 & Rondonópolis - MT & Algodoeiro \\
\hline 56 & Santa Helena - GO & Algodoeiro \\
\hline 57 & Meridiano - SP & Soja \\
\hline 58 & Mira Estrela - SP & Algodoeiro \\
\hline
\end{tabular}

${ }^{\mathrm{a}}$ Cucumis melo L., ${ }^{\mathrm{b}}$ Gossypium hirsutum L.,${ }^{\mathrm{c}}$ Glycine max $(\mathrm{L}$.$) Merril., { }^{\mathrm{d}}$ Sorghum bicolor (L.) Moench, ${ }^{\mathrm{e}}$ Pennisetum americanum (L.) Leeke, ${ }^{\mathrm{f}}$ Zea mays L., ${ }^{\mathrm{g}}$ Annona squamosa L., ${ }^{\mathrm{h}}$ Lycopersicon esculentum Mill., ${ }^{\mathrm{i}}$ Citrus sinensis (L.) Osbeck, ${ }^{\mathrm{j}}$ Daucus carota L., ${ }^{\mathrm{k}}$ Phaseolus vulgaris L., ${ }^{1}$ Carica papaya L.. 
Estudo morfométrico comparativo de 58 populações brasileiras de...

comprimento do corpo (L); 2) distância da região labial à vulva; 3) comprimento do estilete; 4) distância da abertura dos ductos da glândula dorsal esofagiana à base do estilete (DGO); 5) distância da região labial ao poro excretor; 6) comprimento do esôfago; 7) distância da região labial ao término do bulbo basal do esôfago; 8) maior largura do corpo; 9) largura do corpo na região anal; 10) comprimento da cauda e 11) comprimento da porção hialina da cauda. Foram, também, avaliadas as seguintes proporções: 12) a (= comprimento do corpo dividido pela maior largura); 13) b (= comprimento do corpo dividido pelo comprimento do esôfago); 14) b' (= comprimento do corpo dividido pela distância da placa labial ao término do bulbo basal do esôfago); 15) c (= comprimento do corpo dividido pela cauda); 16) c' (= comprimento da cauda dividido pela largura do corpo na região anal); 17) V (= distância da extremidade anterior à vulva, como percentagem do comprimento total do corpo); 18) o (= distância da abertura dos ductos da glândula dorsal esofagiana à base do estilete como percentagem do comprimento do estilete).

As mensurações das imagens digitalizadas foram efetuadas conforme Soares et al. (2003). Utilizando-se o software Statistica ${ }^{\circledR}$ versão 5.0 (StatSoft Inc. 2300 East 14th Street, Tulsa, Ok 74104, EUA) foram efetuadas análises multivariadas de agrupamento e de componentes principais. Para verificação da similaridade entre as populações, adotouse a medida de dissimilaridade denominada distância euclidiana (Sneath \& Sokal, 1973), e como estratégia de agrupamento para identificar a similaridade entre grupos, a "Average Linkage" (UPGMA - Unweighted Pair Group Method with Arithmetic Average), que utiliza a distância média do grupo e expressa o resultado da ordenação das populações em um dendrograma. A análise de agrupamento tem por finalidade reunir, por algum critério de classificação, indivíduos em grupos, de tal forma que exista máxima homogeneidade dentro desses grupos e máxima heterogeneidade entre eles. A análise de componentes principais reduz o conjunto inicial de variáveis em um conjunto menor retendo o máximo da variabilidade espacial inicial em dois eixos ortogonais denominados de componentes principais (autovetores) definidos a partir dos dois maiores autovalores da matriz de covariâncias das variáveis originais.

A amplitude, a média e o desvio padrão foram determinados apenas para as variáveis consideradas mais importantes na identificação de Rotylenchulus spp. (Robinson et al., 1997), ou seja: comprimento do estilete e V. A média dos valores do comprimento do estilete e de $\mathrm{V}$ de cada população foi submetida à chave dicotômica sugerida por Robinson et al. (1997), para identificação das populações.

\section{RESULTADOS E DISCUSSÃO}

\section{Caracteres morfológicos relevantes à taxionomia de Rotylenchulus spp.}

A Figura 1 ilustra os caracteres morfológicos de fêmeas jovens relevantes à identificação de espécies do gênero Rotylenchulus. Para a identificação de espécies do grupo, caracteres morfológicos de fêmeas adultas e presença ou ausência de machos eram tidos como os caracteres de maior peso até 1978. Com efeito, Germani (1978), em estudo morfobiométrico comparativo de três espécies africanas, propôs chave simplificada para identificação das espécies com base em caracteres morfológicos de fêmeas jovens. Robinson et al. (1997) fizeram revisão do gênero e elaboraram chave para identificação das dez espécies válidas do gênero modificando a que havia sido proposta por Germani (1978). Conquanto a forma da cauda em juvenis de segundo estádio (Van Den Berg, 1990) e em fêmeas jovens (Robinson et al., 1997) tenha sido mencionada como um caractere importante para a identificação de algumas espécies do grupo, em uma população de $R$. reniformis examinada no presente estudo (Figura 2), a forma da cauda variou de término estreito arredondado uniforme à truncado e irregular, ocorrendo caso de bifurcação (Figura 2 A), já relatada por Soares et al. (2003). A porção hialina por vezes se mostrava digitada ou espigada e fracamente digitada (Figura $2 \mathrm{H}$ ).

\section{Análise multivariada dos dados de 58 populações de Rotylenchulus spp.}

A análise dos componentes principais mostrou que as variáveis L, distância da extremidade anterior à vulva, comprimento do estilete, DGO, distância da extremidade anterior ao poro excretor, comprimento do esôfago, distância da extremidade anterior ao extremo posterior das glândulas do esôfago, maior largura do corpo, largura do corpo na região anal e as variáveis derivadas b e b' foram as mais discrepantes. O dendrograma relativo à análise multivariada de agrupamentos dos valores dessas variáveis, está graficamente representado na Figura 3 A. As 58 populações foram classificadas em dois grandes grupos, sendo que a média para

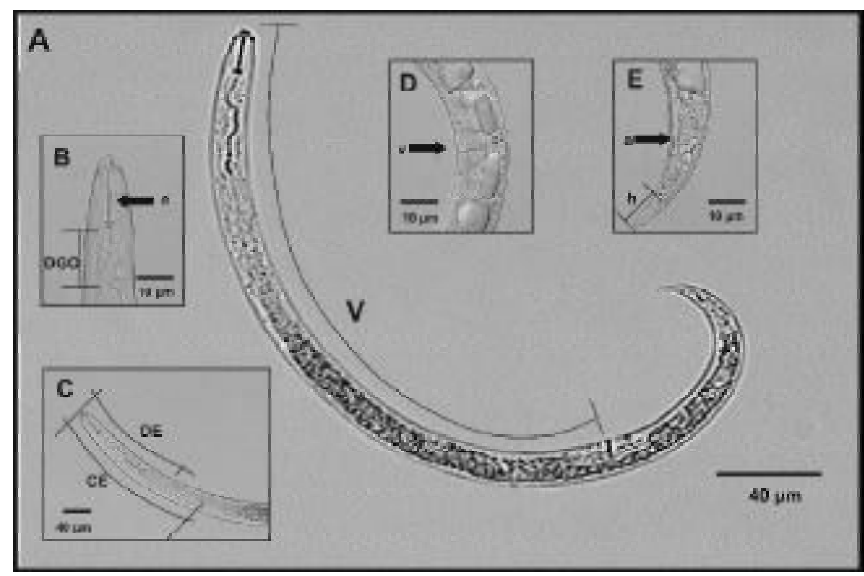

FIG. 1 - Fotomicrografias de fêmea jovem de Rotylenchulus reniformis. A) Fêmea inteira exibindo V; B e C) Região anterior mostrando (e) estilete, (DGO) distância da base do estilete a abertura da glândula dorsal esofagiana, (DE) distância da extremidade anterior ao poro excretor e (CE) comprimento do esôfago; D) Região mediana ilustrando a vulva (v) e E) Região posterior destacando (a) ânus e (h) porção hialina da cauda. 

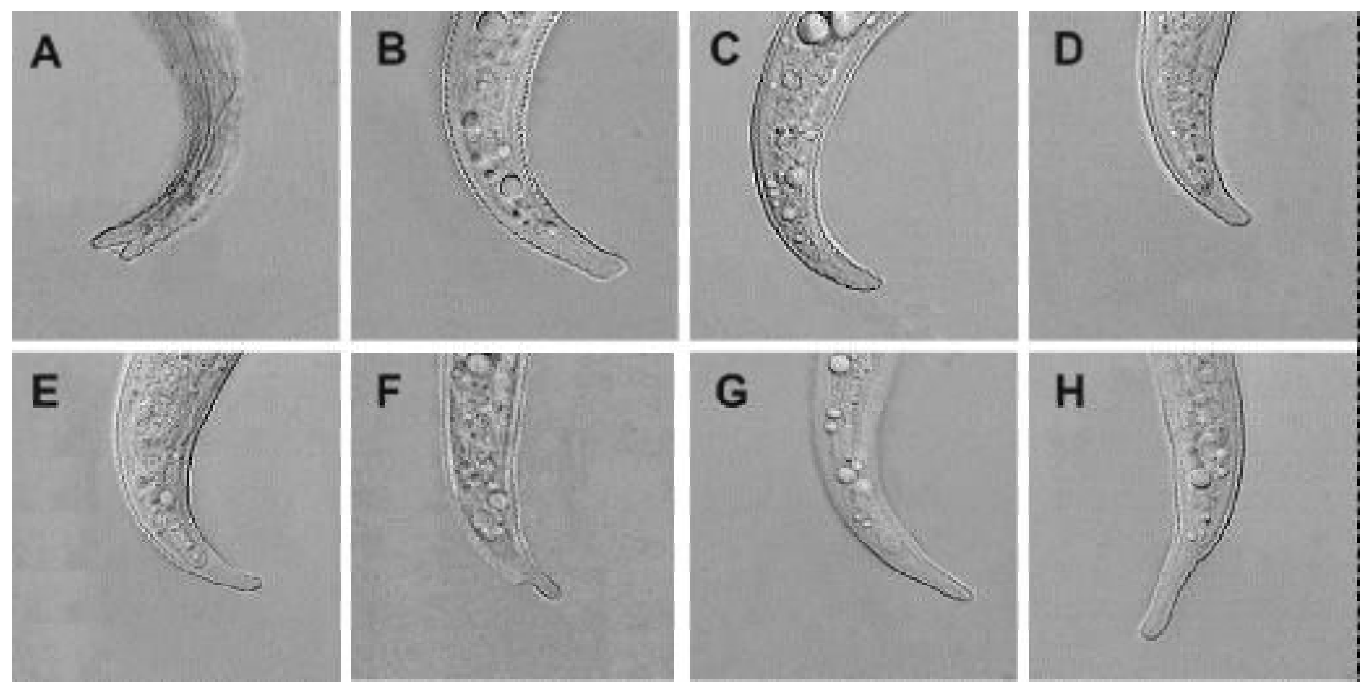

FIG. 2 - Fotomicrografias de caudas de fêmeas jovens da população 43 de Rotylenchulus reniformis, exibindo alta variabilidade morfológica (barra da escala $=10 \mu \mathrm{m}$ para todas as figuras).
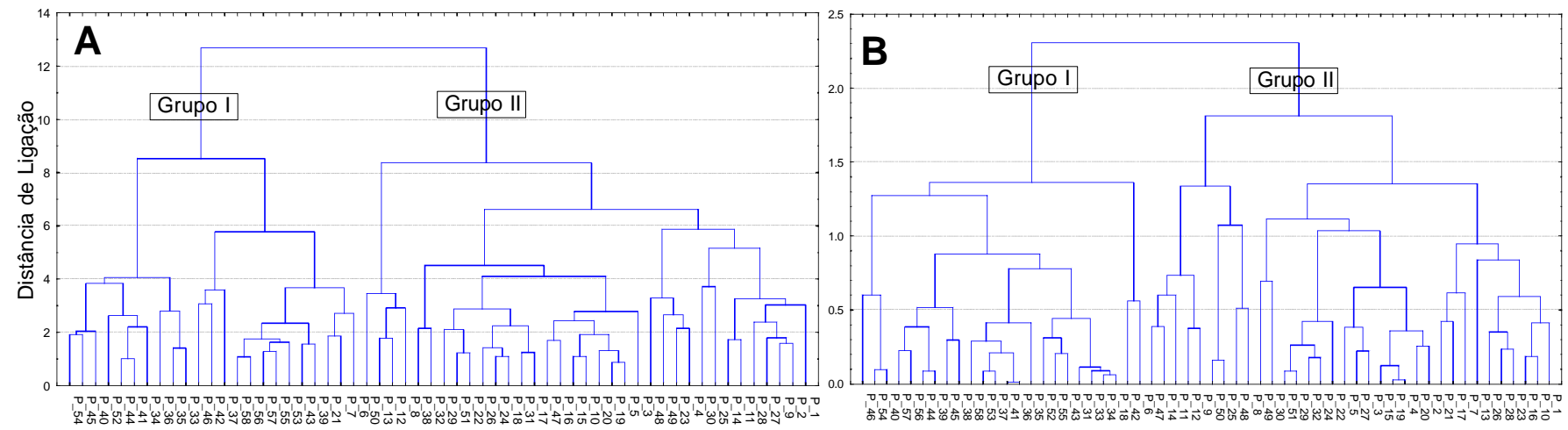

FIG. 3 - Dendrogramas relativos às análises de agrupamentos (“Cluster Analysis”) de fêmeas jovens de 58 populações de Rotylenchulus reniformis com base em valores médios de diferentes variáveis. A) Dendrograma obtido com as variáveis comprimento do corpo (L); distância da placa labial à vulva; comprimento do estilete; DGO; distância da placa labial ao poro excretor; comprimento do esôfago; distância da placa labial ao término do bulbo basal do esôfago; maior largura do corpo; largura do corpo na região anal; b e b'. B) Dendrograma obtido das populações considerando, apenas as variáveis comprimento do estilete e DGO. (os rótulos na abcissa identificam as populações)

o comprimento do estilete no Grupo I (Figura 3 A) foi de 18,2 $\mu$ m com amplitude de variação de 16,5 a 19,1 e a média de $\mathrm{V}$ (\%) de 71,5 com amplitude de 69,2 a 74,0. No Grupo II, a média do comprimento do estilete foi de $16,9 \mu \mathrm{m}$, com amplitude de variação de 15,6 a 18,6 e V (\%) de 71,2, com amplitude de 69,1 a 73,6. Na Figura 3 B, resultados similares foram obtidos efetuando-se a análise das 58 populações com base no comprimento do estilete e na distância do orifício da glândula dorsal esofagiana à base do estilete, apenas. Os valores obtidos no presente estudo estão dentro da amplitude de variação apresentada para essas variáveis entre as diferentes populações de $R$. reniformis do Sudeste do Estados Unidos (Lehman \& Inserra, 1990). Portanto, as 58 populações incluídas no presente estudo são classificadas como $R$. reniformis.

\section{Estudo morfométrico comparativo das 58 populações}

Os dados relativos às mensurações do comprimento do estilete e de V (\%) com suas médias, mínimo, máximo e desvio padrão de dez fêmeas jovens de cada uma das 58 populações estudadas estão incluídos na Tabela 2. Esses dados, submetidos à chave dicotômica proposta por Robinson et. al. (1997), confirmaram que todas as populações estudadas pertencem a $R$. reniformis. A média geral para o valor de $\mathrm{V}$ (\%) foi de 71,4 com amplitude de variação de 61,5 a 80,9. Para o comprimento do estilete foi de $17,6 \mu \mathrm{m}$ com amplitude de variação de 13,7 a 20,7 (Tabela 2). O valor da média para V (\%), obtido da literatura mundial, é de $71(67,0-81,0)$ e do comprimento do estilete é de $17,2 \mu \mathrm{m}(15,0-21,0)$, conforme Lehman \& Inserra (1990). No estudo de 48 populações brasileiras, Soares et al. (2003) obtiveram para V(\%) média 
Estudo morfométrico comparativo de 58 populações brasileiras de...

TABELA 2 - Comprimento do estilete e V (\%) de dez fêmeas jovens de cada uma das 58 populações brasileiras de Rotylenchulus reniformis

\begin{tabular}{|c|c|c|}
\hline $\begin{array}{l}\text { Identificação } \\
\text { da população }\end{array}$ & Estilete & V\% \\
\hline 1 & $16,2^{\mathrm{a}}\left(15,1^{\mathrm{b}}-17,3^{\mathrm{c}}\right) \pm 0,7^{\mathrm{d}}$ & $71,6(69,6-73,3) \pm 1,2$ \\
\hline 2 & $17,0(15,5-18,0) \pm 0,8$ & $71,4(69,8-73,9) \pm 1,3$ \\
\hline 3 & $17,1(15,3-18,0) \pm 0,9$ & $72,0(70,8-75,5) \pm 1,3$ \\
\hline 4 & $17,2(14,7-18,7) \pm 1,1$ & $71,7(69,6-79,6) \pm 3,0$ \\
\hline 5 & $17,0(16,0-17,5) \pm 0,5$ & $71,2(66,3-79,1) \pm 3,4$ \\
\hline 6 & $17,5(16,5-18,8) \pm 0,7$ & $72,6(69,7-80,6) \pm 4,8$ \\
\hline 7 & $16,9(15,3-17,7) \pm 0,9$ & $70,2(60,4-74,9) \pm 4,7$ \\
\hline 8 & $16,0(13,7-17,8) \pm 1,4$ & $71,4(68,3-79,5) \pm 3,3$ \\
\hline 9 & $16,2(15,5-17,9) \pm 0,7$ & $71,6(69,1-74,2) \pm 1,7$ \\
\hline 10 & $16,3(15,4-17,4) \pm 0,7$ & $70,6(68,4-74,5) \pm 1,7$ \\
\hline 11 & $16,2(15,2-17,1) \pm 0,8$ & $70,5(68,9-72,3) \pm 1,1$ \\
\hline 12 & $16,2(15,4-16,9) \pm 0,6$ & $71,1(65,6-77,0) \pm 3,0$ \\
\hline 13 & $15,9(14,8-17,1) \pm 0,9$ & $70,1(62,8-73,1) \pm 2,8$ \\
\hline 14 & $15,8(15,1-16,6) \pm 0,6$ & $71,3(68,7-74,8) \pm 1,8$ \\
\hline 15 & $16,4(15,4-17,9) \pm 0,9$ & $72,5(69,7-76,3) \pm 1,8$ \\
\hline 16 & $16,5(15,3-17,3) \pm 0,7$ & $72,0(69,1-74,9) \pm 1,6$ \\
\hline 17 & $17,0(16,6-17,3) \pm 0,3$ & $71,6(70,2-72,7) \pm 1,1$ \\
\hline 18 & $17,8(16,3-18,7) \pm 0,8$ & $72,6(69,3-80,9) \pm 3,2$ \\
\hline 19 & $16,7(15,0-18,2) \pm 1,0$ & $71,3(67,9-73,3) \pm 1,7$ \\
\hline 20 & $17,4(16,5-18,6) \pm 0,7$ & $72,1(71,0-74,2) \pm 1,1$ \\
\hline 21 & $16,8(15,4-17,8) \pm 0,9$ & $71,9(68,6-73,1) \pm 1,3$ \\
\hline 22 & $17,4(16,4-18,0) \pm 0,6$ & $72,4(70,5-75,5) \pm 1,4$ \\
\hline 23 & $17,1(15,6-18,8) \pm 1,0$ & $71,3(69,2-75,0) \pm 1,6$ \\
\hline 24 & $17,5(16,8-18,3) \pm 0,6$ & $70,8(65,6-74,9) \pm 2,6$ \\
\hline 25 & $16,7(15,5-17,5) \pm 0,8$ & $69,6(65,9-73,1) \pm 2,3$ \\
\hline 26 & $16,6(15,1-18,2) \pm 1,1$ & $71,9(66,5-80,5) \pm 3,7$ \\
\hline 27 & $16,6(14,8-18,2) \pm 1,1$ & $69,6(64,2-71,8) \pm 2,4$ \\
\hline 28 & $16,9(16,0-17,9) \pm 0,6$ & $69,9(66,8-72,4) \pm 2,5$ \\
\hline 29 & $17,5(15,9-19,3) \pm 1,0$ & $70,9(69,4-74,5) \pm 1,5$ \\
\hline 30 & $17,6(16,1-19,6) \pm 0,9$ & $70,8(68,3-72,4) \pm 1,2$ \\
\hline 31 & $17,7(16,5-18,9) \pm 0,9$ & $71,1(67,6-73,4) \pm 1,7$ \\
\hline 32 & $17,6(16,5-18,7) \pm 0,8$ & $70,3(62,0-72,8) \pm 3,2$ \\
\hline 33 & $18,1(16,2-19,1) \pm 1,0$ & $72,3(70,6-75,2) \pm 1,5$ \\
\hline 34 & $18,2(15,7-19,3) \pm 1,1$ & $71,3(69,4-73,5) \pm 1,0$ \\
\hline 35 & $18,2(16,1-19,8) \pm 1,2$ & $72,0(69,9-77,3) \pm 2,6$ \\
\hline 36 & $18,5(16,5-20,2) \pm 1,2$ & $72,5(68,1-77,6) \pm 3,0$ \\
\hline 37 & $18,4(17,2-19,6) \pm 0,8$ & $70,0(65,9-71,7) \pm 1,8$ \\
\hline 38 & $18,5(17,6-19,7) \pm 0,6$ & $71,4(61,5-76,8) \pm 4,1$ \\
\hline 39 & $18,2(16,5-19,8) \pm 1,0$ & $69,0(55,0-75,1) \pm 5,8$ \\
\hline 40 & $18,7(17,7-19,3) \pm 0,5$ & $72,6(70,3-74,3) \pm 1,3$ \\
\hline 41 & $18,6(17,7-19,3) \pm 0,5$ & $70,3(68,2-72,9) \pm 1,4$ \\
\hline 42 & $17,5(16,2-18,6) \pm 0,9$ & $71,1(65,0-75,8) \pm 3,4$ \\
\hline 43 & $18,2(17,1-19,0) \pm 0,6$ & $71,7(67,1-77,4) \pm 3,0$ \\
\hline 44 & $18,6(16,9-19,2) \pm 0,7$ & $71,2(64,3-75,4) \pm 3,5$ \\
\hline 45 & $18,8(17,1-19,7) \pm 0,9$ & $72,0(69,7-76,2) \pm 2,2$ \\
\hline 46 & $19,0(18,5-19,7) \pm 0,5$ & $73,7(69,7-78,1) \pm 2,9$ \\
\hline 47 & $15,8(14,6-17,3) \pm 0,9$ & $70,1(64,4-73,5) \pm 2,6$ \\
\hline 48 & $16,1(15,5-16,6) \pm 0,4$ & $71,6(67,8-75,6) \pm 2,6$ \\
\hline 49 & $17,8(16,8-18,7) \pm 0,8$ & $73,3(71,4-75,1) \pm 1,2$ \\
\hline 50 & $16,8(16,2-18,0) \pm 0,6$ & $70,9(69,1-73,1) \pm 1,4$ \\
\hline 51 & $17,7(17,1-18,4) \pm 0,4$ & $72,5(70,8-75,1) \pm 1,3$ \\
\hline 52 & $18,4(17,0-19,2) \pm 0,7$ & $71,1(68,2-73,4) \pm 1,6$ \\
\hline 53 & $18,6(17,8-19,3) \pm 0,5$ & $71,6(70,5-72,8) \pm 0,9$ \\
\hline 54 & $18,9(18,1-19,4) \pm 0,5$ & $71,0(69,7-74,7) \pm 1,9$ \\
\hline 55 & $18,0(16,8-19,4) \pm 0,9$ & $70,9(68,4-71,9) \pm 1,3$ \\
\hline 56 & $18,8(17,9-19,7) \pm 0,6$ & $71,2(66,6-72,8) \pm 1,9$ \\
\hline 57 & $18,4(17,7-19,5) \pm 0,5$ & $71,5(68,5-74,1) \pm 1,7$ \\
\hline 58 & $18,7(17,8-19,6) \pm 0,6$ & $71,6(68,9-74,3) \pm 1,5$ \\
\hline
\end{tabular}

${ }^{\mathrm{a}}$ Média, ${ }^{\mathrm{b}}$ Valor mínimo, ${ }^{\mathrm{c}}$ Valor máximo, ${ }^{\mathrm{d}}$ Desvio-padrão de 70,8, com amplitude de 55,1 a 84,1, e para o comprimento do estilete, média de 19,2 $\mu$ m com amplitude de 11,6 a 27,8. Conquanto tenha havido diferenças entre os valores máximos e mínimos para essas variáveis, os valores médios estão nas faixas apresentadas para $R$. reniformis.

A amplitude de variação de caracteres morfométricos em populações brasileiras desse nematóide tais como, o comprimento do estilete, V (\%) e a variação na forma da cauda, é maior do que em populações desse nematóide de outras regiões do mundo (Dasgupta et al., 1968; Germani, 1978; Lehman \& Inserra, 1990), confirmando o verificado por Soares et al. (2003).

\section{REFERÊNCIAS BIBLIOGRÁFICAS}

DASGUPTA, D.R. \& SESHADRI, A.R. Races of the reinform nematode, Rotylenchulus reniformis Linford and Oliveira, 1940. Indian Jounal of Nematology 1:21-24. 1971.

DASGUPTA, D.R., RASKI, D.J. \& SHER, S.A. A revision of the genus Rotylenchulus Linford \& Oliveira, 1940. Proceedings of Helminthological Society of Washington 35:169-192. 1968.

GANGULY, S. \& RAMESH, V. Crop-induced morphobiometrical variability in reniform nematode, Rotylenchulus reniformis. I. Mature females and eggs. Annals of Plant Protection Sciences 4:22-28. 1996. (Resumo)

GERMANI, G. Caracteres morpho-biometriques de trois species ouest-africanes de Rotylenchulus Linford and Oliveira, 1940 (Nematoda - Tylenchida). Revue de Nématologie 1:241-250. 1978.

LEHMANN, P.S. \& INSERRA, R.N. Morphometric variation of Rotylenchulus parvus and Rotylenchulus reniformis populations in the southern United States. Soil and Crop Science Society of Florida Proceedings 49:220-226. 1990.

McGAWLEY, E.C. \& OVERSTREET, C. Reproduction and pathological variation in populations of Rotylenchulus reniformis. Journal of Nematology 27:508. 1995.

NAKASONO, K. Studies on morphological and physio-ecological variation of the reniform nematode, Rotylenchulus reniformis Linford \& Oliveira, 1940 with an emphasis on differential geographical distribution of amphimitic and parthenogenetic populations in Japan. Bulletin of the National Institute of Agricultural Sciences 38:63-67. 1983.

PRASADA-RAO,G.M.V., SUDERSHAN, G. \& GANGULY, S. Geographical variations in morphobiometrics of reniform nematode, Rotylenchulus reniformis. Indian Jounal of Nematology 28:56-71. 1998. (Resumo)

ROBINSON, A.F., INSERRA, R.N., CASWELL-CHEN, E.P., VOVLAS, N. \& TROCCOLI, A. Rotylenchulus species: identification, distribution, host ranges, and crop plant resistance. Nematropica 27:127-180. 1997.

SNEATH, P.H.A. \& SOKAL, R.R. Numerical taxonomy. San Francisco. W. H. Freeman Co. 1973.

SOARES, P.L.M., SANTOS, J.M. dos \& LEHMAN, P.S. Estudo morfométrico comparativo de populações de Rotylenchulus reniformis (Nemata: Rotylenchulinae) do Brasil. Fitopatologia Brasileira 28:292-297. 2003

SUDERSHAN, G., RAMESH, V. \& GANGULY, S. Crop-induced 


\section{P.L.M. Soares et al.}

morphobiometrical variability in reniform nematode, Rotylenchulus reniformis. II. Young females, males and juveniles. Annals of Plant Protection Sciences 5:75-89. 1997. (Resumo)

SUYADI, M.S. Quantitative method in nematode species identification of the genus Rotylenchulus. Buletin Budidaya Pertanian,
Indonésia 2:1-10. 1997. (Resumo)

VAN DEN BERG, E. Two new and one known species of the Tylenchoidea (Nemata) from Southern Africa. Phytophylactica 22:2334. 1990. 